\title{
Monocapas de ácidos grasos. III. Ácidos palmítico, láurico y oleico sobre disoluciones acuosas que contienen solutos con grupos funcionales alcohólicos.
}

\author{
Por J. M. Rodríguez Patino, J. de la Fuente Feria y C. Gómez Herrera. \\ Departamento de Ingeniería Química. Facultad de Química. \\ Universidad de Sevilla. C/. Prof. García González, s/n. \\ 41012 - SEVILLA (España). Fax: 4624952.
}

\section{RESUMEN}

Monocapas de ácidos grasos. III. Ácidos palmítico, láurico y oleico sobre disoluciones acuosas que contienen solutos con grupos funcionales alcohólicos.

Se ha estudiado la influencia que ejercen la longitud y la insaturación de la cadena acilica sobre las características de las monocapas de ácidos grasos esparcidas sobre medios acuosos que contienen etanol, glicerina, glucosa o sacarosa, utilizando una balanza de superficie de tipo Langmuir.

La estabilidad de la monocapa es función de la longitud de la cadena acilica y de la presencia de insaturación. En general, los mismos factores que disminuyen la estabilidad de la monocapa pueden favorecer las transiciones hacia configuraciones con estructuras más expandidas.

PALABRAS-CLAVE: Balanza de superficie - Disolución acuosa de moléculas hidroxillicas - Láurico (ácido) - Oleico (ácido) - Palmítico (ácido).

\section{SUMMARY}

Monolayers of fatty acids. III. Palmitic, Lauric and Oleic Acids on aqueous solutions containig solutes with alcoholic functional groups.

The influence exerted by the length or the insaturation of acyl chain on characteristics of fatty acid monolayers spread on aqueous solutions containing ethanol, glycerol, glucose or sucrose, is studied using a Langmuir type surface balance.

The monolayer stability is function of acyl chain length and presence of insaturarion. Generally, the factors decreasing monolayer stability can help transformations towards configurations with more expanded structures.

KEY-WORDS: Aqueous solution of hydroxylic molecules Lauric (acid) - Oleic (acid) - Palmitic (acid) - Surface balance.

\section{INTRODUCCIÓN}

En las aplicaciones industriales de los aditivos alimentarios utilizados como emulsionantes o estabilizadores de interfases, las características de las monocapas formadas en la interfase aire-medio acuoso poseen un interés práctico derivado de su influencia sobre la estabilidad y propiedades de espumas y emulsiones (1-4), o sobre alguna de las etapas de procesado o aplicación de determinados alimentos (5) (6).

Además de la variación que se produce en las propiedades físicas del sistema monocapa-subfase, al variar los componentes de la última y su concentración (7) (8) existe otro factor no de menos importancia que incide sobre la disposición de las moléculas en la monocapa; dicho factor es el tipo de interacción que exista entre las moléculas de lípidos y entre ellas y la subfase.

Para una monocapa de un lípido insoluble un análisis general de las fuerzas superficiales debe considerar las interacciones lípido-lípido y lípidosubfase. Los métodos clásicos para evaluar estas fuerzas en sistemas condensados han consistido en la medida de las energías internas del sistema. No obstante, la baja presión de vapor de la mayoría de los lípidos que forman monocapas condensadas introduce una limitación práctica en la medida directa de la energía interna (9), aunque se han desarrollado diversos métodos alternativos (10) (11). Uno de estos métodos (11) utiliza el hecho de que para series homólogas de compuestos alifáticos normales existe una constante, independiente de la naturaleza del grupo polar, que contempla el incremento de la energía libre con la compresión de la monocapa cuando aumenta la longitud de la cadena hidrocarbonada. Este resultado es consistente con el principio de Langmuir de independencia de la acción superficial (12) (13). Según este principio las monocapas condensadas pueden considerarse constituidas de dos partes: la parte superior correspondiente a la cadena hidrocarbonada, que se comporta como una fase separada con una energía interna característica, y el dominio del grupo polar que también contribuye independientemente a la presión superficial de la monocapa. 
Las fuerzas intermoleculares que actúan en una monocapa se pueden incluir en alguna de las siguientes categorías: a) Interacciones de largo alcance entre las cabezas polares; b) Interacciones de corto alcance entre los átomos de carbono de las cadenas hidrocarbonadas situados a mayor distancia del grupo polar; c) Atracciones de largo alcance o interacciones de Van der Waals entre las cadenas; d) Interacciones de largo alcance entre las cadenas tendentes a introducir un desorden en el sistema. De hecho, se han propuesto una serie de modelos teóricos para explicar las estructuras de las monocapas según las interacciones entre las moléculas (14-19). Estos modelos están basados en la importancia relativa de las fuerzas intermoleculares antes citadas, de las cuales las interacciones de Van der Waals están contempladas en todos. El efecto de la longitud de la cadena se ha incluido sólo en contadas ocasiones, aunque Marcelja (20) y Caillé (14) (21) han investigado su importancia en función de la dependencia entre el punto de fusión del lípido y el tipo de cadena hidrocarbonada. Recientemente, Pekalski (22) ha propuesto un modelo en el cual determina la relación entre los efectos de la longitud de la cadena hidrocarbonada de un lípido sobre el diagrama de fases, considerando la intervención de las cuatro interacciones citadas.

Según las experiencias efectuadas en balanza de superficie, de las que estos estudios iniciales sobre ácidos grasos (23) (24) pueden servir de referencia para el estudio de sistemas más complejos y de mayor interés industrial, como los constituidos por los monoglicéridos y ésteres de los propios ácidos grasos con polioles, las características de estas monocapas se resumen en la estructura que adoptan las moléculas que las constituyen y en su solubilidad en el medio acuoso, según las condiciones de realización del proceso. El objetivo del presente trabajo se centra en el estudio de la influencia de la longitud de la cadena hidrocarbonada y de la presencia de insaturación en la misma sobre las características de las monocapas.

\section{MATERIALES Y MÉTODO}

Las isotermas (curvas presión de esparcimiento, $\pi$, frente al área por molécula, A) se han obtenido en una balanza de superficie comercial tipo Langmuir, equipada con un sistema de medida continuo y registrador. La temperatura de la bandeja que contiene la monocapa y la subfase se mantiene constante durante cada operación mediante la circulación de agua desde un termostato, con una sensibilidad en el control de temperatura de $\pm 0,01-0,03^{\circ} \mathrm{C}$. La forma de operar se ha descrito en un trabajo anterior (23).

Los productos usados son de calidad analítica y no han sido sometidos a purificación posterior: ácido palmítico (Merck, Cat. 800508), ácido laúrico (Merck, Cat. 805333), ácido oleico (Reagente, $\mathrm{BDH}$ ), etanol (Merck, Cat. 971), glicerina (Merck, Cat. 4093), glucosa (Merck, Cat. 8346), sacarosa (Merck, Cat. 7654). El agua fue purificada mediante una unidad de filtración Millipore (Mille $Q^{T M}$ ). Los lípidos fueron esparcidos sobre la subfase previa disolución en benceno (Merck, Cat. 1783). La falta de actividad superficial de los componentes de la subfase y del benceno usado se ha comprobado experimentalmente.

\section{RESULTADOS Y DISCUSIÓN}

\subsection{Monocapas de ácido palmítico.}

\section{Monocapas de ácido palmítico esparcidas sobre agua desionizada.}

Las experiencias referidas al ácido palmítico como constituyente de la monocapa se inician esparciendo el lípido sobre agua desionizada. Los resultados obtenidos al variar la temperatura del sistema se representan en la figura 1. De estas experiencias se deduce lo siguiente:

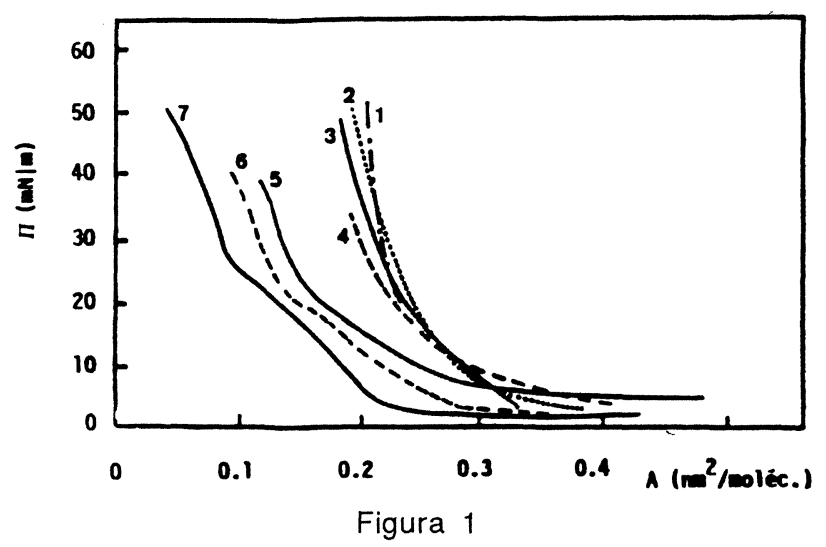

Isotermas de compresión presión superficial-área por molécula de ácido palmítico esparcido sobre agua desionizada. Temperaturas. $1: 5^{\circ} \mathrm{C}, 2: 10-15^{\circ} \mathrm{C}, 3: 20^{\circ} \mathrm{C}, 4: 25^{\circ} \mathrm{C}$, 5: $30^{\circ} \mathrm{C}, 6: 35^{\circ} \mathrm{C}, 7: 40^{\circ} \mathrm{C}$.

* A la menor temperatura de operación $\left(5^{\circ} \mathrm{C}\right)$, la isoterma registrada muestra la existen- 
cia de una transición en la estructura de la monocapa de tipo líquido condensado $=$ sólido. Esta transición se produce a la presión de $23,4 \mathrm{mN} / \mathrm{m}$ y el valor del área molecular, que se deduce por la prolongación del segmento característico de la estructura sólida de la monocapa, es de $0,244 \mathrm{~nm}^{2} /$ molécula.

* Al incrementarse la temperatura por encima de los $5^{\circ} \mathrm{C}$ se observan dos hechos que repercuten de forma distinta sobre las estructuras que adoptan las moléculas de la monocapa:

** En el intervalo comprendido entre $10^{\circ} \mathrm{C}$ y $20^{\circ} \mathrm{C}$ la monocapa adquiere una estructura de líquido condensado si bien, como consecuencia posiblemente de la disolución del lípido en la subfase, las isotermas registradas no muestran una incidencia significativa de la variable independiente sobre la solicitud de área por las moléculas, aunque sí es observable una disminución de la presión de colapso de la monocapa con el citado incremento de la temperatura.

** En el intervalo comprendido entre $30^{\circ} \mathrm{C}$ y $40^{\circ} \mathrm{C}$, la monocapa posee las estructuras de líquido condensado y de líquido expandido en función del valor de la presión de compresión, es la típica estructura intermedia (I) definida por Harkins (25). Aunque la monocapa mantiene esta estructura expandida, la presión de colapso se incrementa al aumentar la temperatura, una característica inversa a la observada a temperaturas inferiores. Además, al aumentar el valor de esta variable se produce una traslación de las isotermas registradas hacia menores solicitudes de área, un hecho cuya justificación más coherente ha de atribuirse al incremento de la solubilidad de las moléculas de la monocapa en el sentido de las temperaturas crecientes, según las razones expuestas en artículos anteriores (23) (24).

\section{Monocapas de ácido palmítico esparcidas sobre disolución acuosa de etanol}

Las monocapas esparcidas sobre una disolución de etanol a $0,5 \mathrm{~mol} / \mathrm{L}$ como subfase (figura 2) poseen una estructura sólida, independientemente de la temperatura de operación. No obstante, la presión de colapso aumenta sensiblemente con el incremento de la variable adquiriendo unos valores, a las mayores temperaturas, superiores a los observados con el mismo lípido esparcido sobre agua desionizada. Además, la solubilidad de la monocapa se incrementa al aumentar la temperatura en mayor proporción como consecuencia de la presencia de etanol en la subfase. Véase que el área por molécula obtenida por extrapolación de cada isoterma se reduce en un porcentaje que supera el $70 \%$ al modificarse la temperatura desde $5^{\circ} \mathrm{C}$ hasta $40^{\circ} \mathrm{C}$ (tabla I). Los resultados obtenidos pueden ser explicados en función de la existencia de interacciones en la cadena hidrocarbonada, que se traduce en la presencia de fuertes repulsiones en la monocapa como para mantener la estructura sólida independientemente de la temperatura (26), e interacciones entre el grupo polar del lípido y las moléculas superficiales de la disolución alcohólica, que se traduce en una modificación de la solubilidad del mismo con la temperatura (27-29).

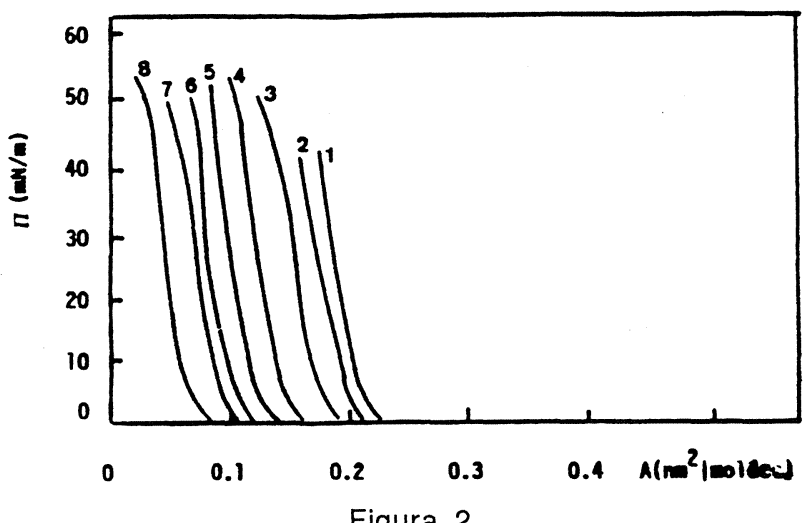

Isotermas de compresión presión superficial-área por molécula de ácido palmítico esparcido sobre disolución acuosa de etanol a $0.5 \mathrm{~mol} / \mathrm{L}$. Temperaturas. $1: 5^{\circ} \mathrm{C}, 2: 10^{\circ} \mathrm{C}, 3$ : $15^{\circ} \mathrm{C}, 4: 20^{\circ} \mathrm{C}, 5: 25^{\circ} \mathrm{C}, 6: 30^{\circ} \mathrm{C}, 7: 35^{\circ} \mathrm{C}, 8: 40^{\circ} \mathrm{C}$.

\section{Tabla I}

Presión de colapso y área límite en función de la temperatura para monocapas de ácido palmítico sobre disolución de etanol a $0,5 \mathrm{~mol} / \mathrm{L}$.

\begin{tabular}{|c|c|c|}
\hline $\mathrm{T}\left({ }^{\circ} \mathrm{C}\right)$ & $\pi_{c}(\mathrm{mN} / \mathrm{m})$ & $A_{\text {tin }}\left(\mathrm{nm}^{2} /\right.$ moléc. $)$ \\
\hline 5 & $28^{\prime} 0$ & 0.206 \\
\hline 10 & $36 \cdot 2$ & 0.200 \\
\hline 15 & $52^{\prime} 4$ & 0.180 \\
\hline 20 & $54^{\prime} 0$ & $-\quad 0.145$ \\
\hline 25 & $53 \cdot 5$ & 0.116 \\
\hline 30 & $54^{\prime} 0$ & 0.091 \\
\hline 35 & $55^{\prime} 7$ & 0.096 \\
\hline 40 & $55^{\prime} 5$ & 0.055 \\
\hline
\end{tabular}




\section{Monocapas de ácido palmítico esparcidas sobre disolución acuosa de glicerina}

En la figura 3 se representan las isotermas registradas correspondientes a las compresiones de monocapas de ácido palmítico esparcidas sobre una disolución acuosa de glicerina a $0,3 \mathrm{~mol} / \mathrm{L}$ como subfase. Desde un punto de vista cualitativo puede afirmarse que, como consecuencia de la presencia de las moléculas de glicerina en la subfase, la variación de la temperatura del sistema ejerce una influencia sobre las interacciones de las cadenas hidrocarbonadas del lípido entre sí que es significativamente superior a su incidencia sobre la estabilidad de la monocapa. En efecto, a partir de los resultados obtenidos se deduce la existencia de un polimorfismo, función de la temperatura de operación, que se manifiesta en la adopción, por las moléculas que constituyen la monocapa, de una estructura líquido condensado que se transforma en sólida tras la compresión, a las menores temperaturas $\left(5-10^{\circ} \mathrm{C}\right)$. A temperaturas superiores las moléculas de la monocapa adquieren una configuración de líquido expandido, que se potencia al aumentar la temperatura. Además, la función existente entre la presión de colapso de la monocapa y la temperatura es decreciente en el intervalo estudiado, según se expone en la tabla II. Estos resultados pueden ser la consecuencia de la existencia de una inestabilidad de la monocapa por disolución de la misma en la subfase. Como es sabido, una reducción de las fuerzas de cohesión de Van der Waals entre las moléculas de la monocapa suele traducirse en un descenso de la presión de colapso (8) (30) e, incluso, condicio-

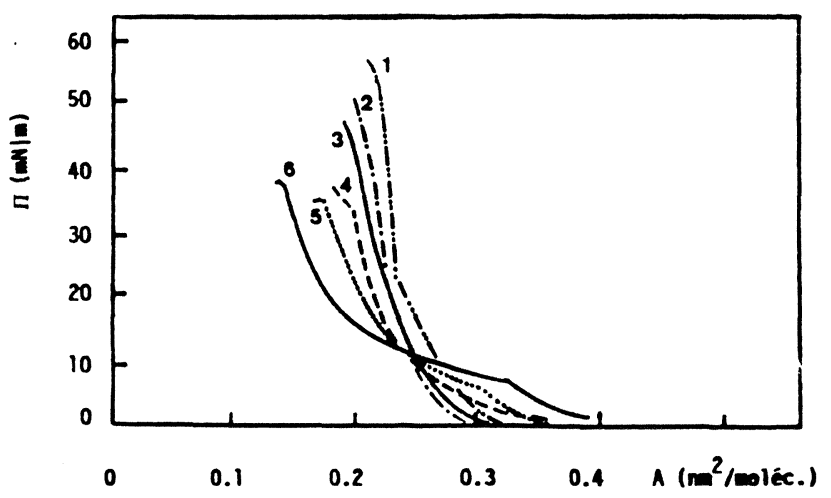

Figura 3

Isotermas de compresión presión superficial-área por molécula de ácido palmítico esparcido sobre disolución acuosa de glicerina a $0.3 \mathrm{~mol} / \mathrm{L}$. Temperaturas. $1: 5-10^{\circ} \mathrm{C}$,

2: $15^{\circ} \mathrm{C}, 3: 20-25^{\circ} \mathrm{C}, 4: 30^{\circ} \mathrm{C}, 5: 35^{\circ} \mathrm{C}, 6: 40^{\circ} \mathrm{C}$. nar la propia existencia de la monocapa (8). Así pues, la expansión que se produce en la estructura de la monocapa al aumentar la temperatura no se manifiesta en un incremento paralelo de la solicitud de área molecular como consecuencia de la existencia de una cierta solubilidad del lípido en la subfase, lo que justifica que las distintas isotermas registradas estén situadas en el diagrama $\pi$ A de la figura 3 en una banda estrecha.

Tabla II

Presión de colapso de monocapas de ácido palmítico esparcidas sobre disoluciones acuosa de glicerina a 0,3 $\mathrm{mol} / \mathrm{L}$.

\begin{tabular}{|ll|}
\hline $\mathrm{T}\left({ }^{\circ} \mathrm{C}\right)$ & $\pi_{c}(\mathrm{mN} / \mathrm{m})$ \\
\hline 5 & $57 \cdot 5$ \\
10 & $57^{\prime} 5$ \\
15 & 50 \\
20 & $47 \cdot 6$ \\
25 & 43 \\
30 & $33 \cdot 7$ \\
35 & $36 \cdot 5$ \\
40 & $39 \cdot 5$ \\
\hline
\end{tabular}

\section{Monocapas de ácido palmítico esparcidas sobre disolución acuosa de glucosa}

Las monocapas de ácido palmítico esparcido sobre una disolución acuosa de glucosa a $0,1 \mathrm{~mol} / \mathrm{L}$ presentan una estructura claramente expandida, según se muestra en la figura 4. Esta estructura expandida se manifiesta incluso a valores elevados de la presión de compresión, de $40 \mathrm{mN} / \mathrm{m}$ a la temperatura de $25^{\circ} \mathrm{C}$, en las experiencias realizadas con este sistema. Desde otro punto de vista, el incremento esperable en la expansión de las moléculas de la monocapa por aumento de la temperatura se compensa prácticamente por el aumento de la solubilidad de las mismas. Como característica fundamental de estas monocapas puede destacarse su baja compresibilidad, $[-(1 / A)$ $(\mathrm{d} \pi / \mathrm{dA})]$, independientemente del valor de la temperatura de operación o de la presión ejercida sobre las moléculas que la constituyen. 


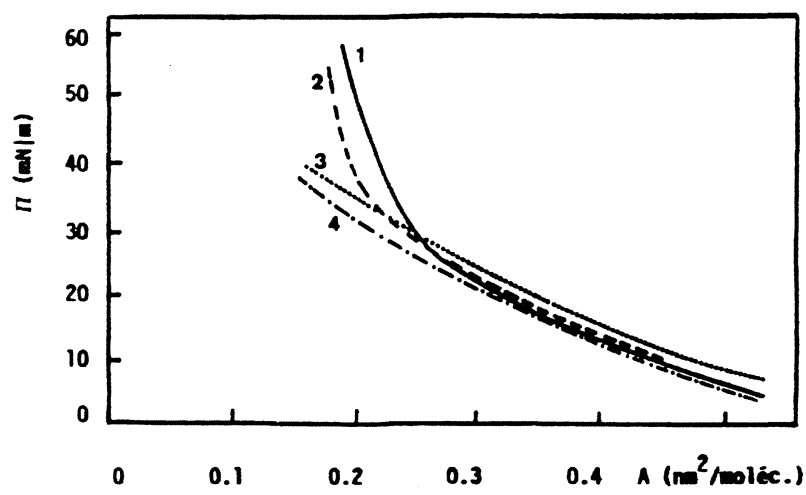

Figura 4

Isotermas de compresión presión superficial-área por molécula de ácido palmítico esparcido sobre disolución acuosa de glucosa a $0.1 \mathrm{~mol} / \mathrm{L}$.

Temperaturas. $1: 5^{\circ} \mathrm{C}, 2: 10^{\circ} \mathrm{C}, 3: 20^{\circ} \mathrm{C}, 4: 25^{\circ} \mathrm{C}$.

\section{Discusión global}

Una discusión global de los resultados obtenidos con monocapas de ácido palmítico se puede resumir en los siguientes puntos:

* La temperatura y la composición de la subfase ejercen una influencia decisiva sobre la estructura de la monocapa:

** Un aumento de temperatura produce una expansión en la estructura de la monocapa, manifestándose transiciones hasta los estados gaseoso o líquido expandido, según sea el soluto de las disoluciones utilizadas como subfases. Estas transiciones siguen el orden indicado en la tabla III.

\section{Tabla III}

Interdependencia entre la estructura de monocapas de ácido palmítico y la temperatura, en función de la composición de la subfase.

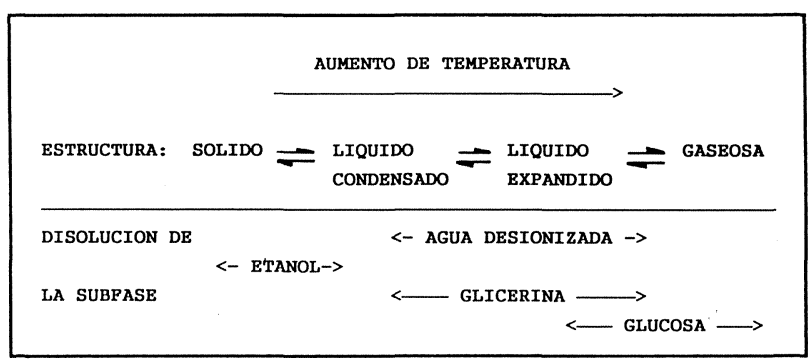

** Las monocapas esparcidas sobre etanol constituyen una excepción del comportamiento citado previamente, observándose la persistencia de la estructura sólida inde- pendientemente de la temperatura del sistema.

** En las monocapas con estructuras sólida o de líquido condensado, un aumento de temperatura produce un incremento en el valor de la presión de colapso. En las monocapas con estructuras de líquido expandido o gaseosa se observa el fenómeno inverso, excepto en el sistema con subfase de agua desionizada en el cual la solubilidad de la monocapa juega un papel fundamental a las temperaturas medias y elevadas.

* La temperatura y la composición de la subfase ejercen una influencia desigual sobre la solubilidad de la monocapa:

** En general, un aumento de temperatura produce un incremento de la solubilidad de la monocapa que es cuantitativamente más importante a valores de la variable superiores a $20^{\circ} \mathrm{C}$

* En función de los desplazamientos de las isotermas registradas, como consecuencia del aumento de temperatura, hacia menores solicitudes de áreas, un fenómeno que se ha asociado a la existencia de rdisolución de moléculas de la monocapa, la composición de la subfase reduce la solubilidad de la misma en el siguiente orden:

$\mathrm{H}_{2} \mathrm{O}>\mathrm{ETANOL}>$ GLICERINA > GLUCOSA

\subsection{MONOCAPAS DE ÁCIDO LÁURICO}

El ácido láurico es el que posee la cadena hidrocarbonada lineal con menor número de átomos de carbono de todos los estudiados en la misma serie homóloga. Ello aconseja centrar la atención de las experiencias en la estabilidad de la monocapa $y$, en efecto, a partir de los estudios previos realizados con este lípido, se ha creído conveniente efectuar su esparcimiento a la menor temperatura de operación $\left(5^{\circ} \mathrm{C}\right)$.

\section{Monocapas de ácido láurico esparcidas sobre agua desionizada}

Las experiencias realizadas con el ácido láurico esparcido sobre agua desionizada se resumen en la figura 5. Las compresiones de la monocapa a la temperatura de $5^{\circ} \mathrm{C}$ se repiten tres veces, produciéndose una traslación de las isoter- 
mas hacia la zona de menores requerimientos de área por molécula. Este hecho podría ser atribuido a la existencia de un proceso de disolución de la monocapa; sin embargo, las isotermas registradas poseen dos características cuyos efectos se podrían contradecir con la posible pérdida de moléculas de soluto desde la superficie: la monocapa se hace menos expandida y la presión de colapso disminuye al repetirse las compresiones. Estos son dos fenómenos que están directamente relacionados con las interacciones entre las cadenas hidrocarbonadas y entre el grupo polar y las moléculas superficiales de la subfase (8). Es posible, no obstante, que tras la primera compresión se haya producido el colapso de la monocapa y que las diferencias entre las isotermas correspondientes a compresiones sucesivas sean debidas a un proceso de relajación hasta la adopción, por las moléculas que constituyen la monocapa, de una estructura estable con una "reducción" ficticia del área molecular (8).

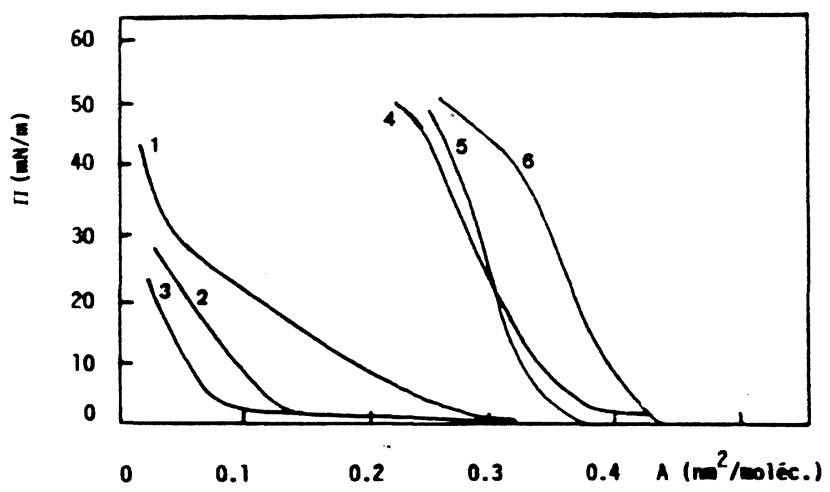

Figura 5

Isotermas de compresión presión superficial-área por molécula de ácido láurico esparcido sobre agua desionizada.

Temperaturas. $1: 5^{\circ} \mathrm{C}\left(1 . .^{3}\right.$ compresión), 2: $5^{\circ} \mathrm{C}\left(2 .{ }^{3}\right.$ compresión), 3: $5^{\circ} \mathrm{C}$ (3. ${ }^{3}$ compresión), 4: $20^{\circ} \mathrm{C}\left(1 . .^{\mathrm{a}}\right.$ compresión), $5: 20^{\circ} \mathrm{C}\left(2 .^{a}\right.$ compresión), $6: 40^{\circ} \mathrm{C}$

Las isotermas registradas a la temperatura de $20^{\circ} \mathrm{C}$ parecen confirmar la ausencia de disolución significativa de la monocapa a la temperatura de $5^{\circ} \mathrm{C}$. En efecto, véase que a la temperatura de $20^{\circ} \mathrm{C}$ la monocapa posee la misma estructura de líquido condensado, pero con mayores requerimientos de área por molécula. Además, resulta interesante destacar que durante la compresión no se registra la típica inflexión característica del colapso en otros lípidos (8) sino que, superado el valor de $50 \mathrm{mN} / \mathrm{m}$, la presión se hace independiente del área por molécula de la monocapa.
Para profundizar en estos puntos se realizan distintos ensayos, manteniéndose constante la temperatura en $20^{\circ} \mathrm{C}$

* Se efectúa un ensayo completo de compresión pero manteniéndose estática la barrera móvil, durante un período de un minuto, en las posiciones correspondientes a un número par de nanómetros cuadrados/ molécula en el valor del área ocupada por las moléculas de la monocapa. Cada vez que se detiene la barrera móvil se produce un descenso en el valor de la presión $\left(\pi^{\prime}\right)$ que depende de la solicitud de área por las moléculas de la monocapa, según se indica en la tabla IV. Este descenso en el valor de la presión, que es distinto al que caracteriza la llegada al punto de colapso ya que al reiniciar la experiencia el sistema vuelve a la misma posición de partida (8), parece indicar que, si se ha producido un colapso previo a la temperatura de $5^{\circ} \mathrm{C}$, la nueva estructura adquirida por la monocapa es estable al menos durante el período correspondiente a las experiencias realizadas. Esta isoterma (curva 4 en la figura) se diferencia de la registrada con una compresión continua de la monocapa por poseer una menor pendiente (curva 5 en la figura). Es decir, las moléculas que la constituyen adquieren una configuración más expandida.

Tabla IV

\begin{tabular}{c} 
A $\left(\mathrm{nm}^{2} / \mathrm{moléc}\right)$ \\
\hline $0 \cdot 24$ \\
$0 \cdot 26$ \\
$0 \cdot 28$ \\
$0 \cdot 30$ \\
$0 \cdot 32$ \\
$0 \cdot 34$ \\
$0 \cdot 36$ \\
$0 \cdot 38$ \\
$0 \cdot 40$
\end{tabular}

\begin{tabular}{l}
$\pi^{\prime}(\mathrm{mN} / \mathrm{m})$ \\
\hline $7 \cdot 35$ \\
$7 \cdot 25$ \\
$7 \cdot 25$ \\
$6 \cdot 5$ \\
$5 \cdot 0$ \\
$3 \cdot 4$ \\
$2 \cdot 5$ \\
$1 \cdot 25$ \\
$0 \cdot 5$
\end{tabular}

* Una posterior compresión con un valor de la velocidad de avance de la barrera móvil mitad del fijado en experiencias anteriores (1'15 cm/min), conduce a un registro que se identifica con la existencia de una es- 
tructura ligeramente más expandida de las moléculas de la monocapa: los valores del área molecular correspondientes a las velocidades de 3 '3 y $1^{\prime \prime} 15 \mathrm{~cm} / \mathrm{min}$ y a la presión de $50 \mathrm{mN} / \mathrm{m}$ son 0'244 y $0^{\prime} 23 \mathrm{~nm}^{2 /}$ moléc., respectivamente.

A la temperatura de $40^{\circ} \mathrm{C}$ se realiza una primera compresión de la monocapa deduciéndose de la isoterma correspondiente una estructura en las moléculas más expandida y con mayores requerimientos de área, si se la compara con los registros a temperaturas inferiores. Al igual que a estas temperaturas, una vez superado el valor de $52 \mathrm{mN} / \mathrm{m}$, la presión es independiente del área ocupada por las moléculas de la monocapa y la isoterma registrada se hace horizontal en el intervalo comprendido entre 0,24 y $0,05 \mathrm{~nm}^{2} /$ moléc.

Finalmente, y a la misma temperatura de $40^{\circ} \mathrm{C}$, se procede a la realización de distintas compresiones a presión constante, fijándose los valores de la variable en distintos porcentajes de su valor máximo total característico para cada sistema. Los resultados obtenidos se resumen en la Tabla $V$. Como es usual en experiencias con monocapas, las moléculas de estas adquieren configuraciones más condensadas al aumentar la presión superficial.

Tabla $\mathrm{V}$

Solicitud de área por molécula a $40^{\circ} \mathrm{C}$ correspondiente a una monocapa de ácido láurico esparcida sobre agua desionizada, en experiencias realizadas a presión constante.

\begin{tabular}{|c|c|c|c|}
\hline $\begin{array}{c}\text { Presión } \\
\text { seleccionada } \\
\text { (₹P) }\end{array}$ & $\begin{array}{l}\text { Presión } \\
\text { registrada } \\
(\mathrm{mN} / \mathrm{m})\end{array}$ & $\begin{array}{l}\text { Presión total } \\
\text { equivalente } \\
(\mathrm{mN} / \mathrm{m})\end{array}$ & $\begin{array}{l}\text { Solicitud } \\
\text { de área } \\
\text { (nñ²/moléc.) }\end{array}$ \\
\hline 30 & 18.5 & 61.6 & 0.157 \\
\hline 50 & 29.5 & 59.0 & 0.146 \\
\hline 60 & $35 \cdot 75$ & 59.6 & 0.146 \\
\hline 80 & $47 \cdot 75$ & 59.7 & 0.142 \\
\hline 90 & $53 \cdot 75$ & $59 \cdot 7$ & 0.133 \\
\hline \multirow[t]{2}{*}{100} & $59 \cdot 75$ & $59 \cdot 75$ & 0.045 \\
\hline & & $\pi=59.9$ & \\
\hline
\end{tabular}

\section{Monocapas de ácido láurico esparcidas sobre disolución acuosa de ácido cítrico}

Las experiencias previas realizadas con ácido láurico esparcido sobre disoluciones acuosas de etanol y glicerina muestran la falta de estabilidad de las monocapas, derivada presumiblemente de la existencia de disolución de la misma, lo cual dificulta extraordinariamente abordar su estudio. Se ha creído conveniente completar el estudio con este lípido realizando una serie de experiencias espar- ciendo el lípido sobre una disolución ácida, que impida su ionización en la interfase. Como tal se han utilizado una disolución acuosa de ácido cítrico a $0,1 \mathrm{~mol} / \mathrm{L}$. Los resultados obtenidos, a la temperatura de $5^{\circ} \mathrm{C}$, se representan en la figura 6 . Como características fundamentales de la monocapa se pueden destacar la práctica ausencia de difusión desde la interfase (las curvas registradas tras repetidas compresiones son esencialmente las mismas) y la baja compresibilidad de la monocapa. Véase que, incluso a bajas temperaturas, las moléculas de la monocapa adquieren una estructura altamente expandida (prácticamente coincidente con una estructura gaseosa) y con un valor de la presión de colapso de $42 \mathrm{mN} / \mathrm{m}$, aproximadamente.

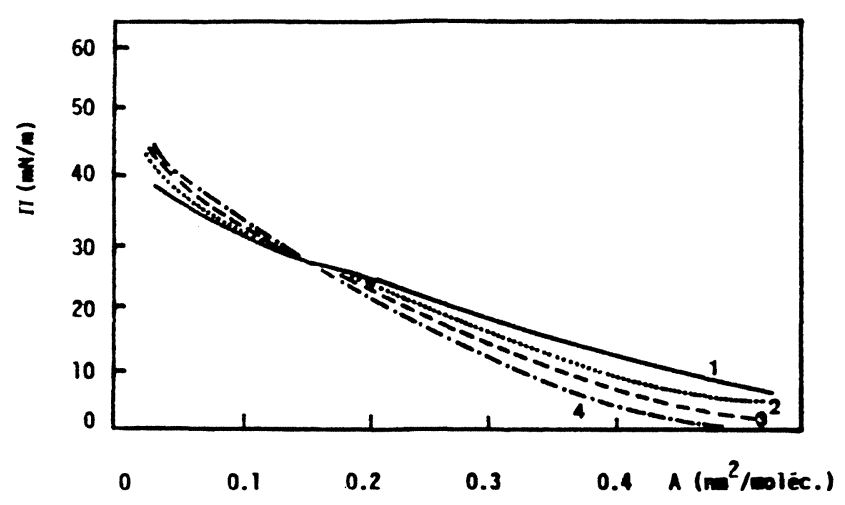

Figura 6

Isotermas de compresión presión superficial-área por molécula de ácido láurico esparcido sobre disolución acuosa de ácido cítrico a $0.1 \mathrm{~mol} / \mathrm{L}$.

Temperatura: $5^{\circ} \mathrm{C}$ (numeradas por orden de compresión).

\subsection{Monocapas de ácido oleico}

El ácido oleico se diferencia de los estudiado previamente por la presencia de un doble enlace en la cadena hidrocarbonada, lo cual puede alterar significativamente las interacciones entre las mismas.

\section{Monocapas de ácido oleico esparcidas sobre agua desionizada}

Las experiencias realizadas con ácido oleico sobre agua desionizada, cuyos resultados se resumen en la representación de la figura 7 , se 
inician esparciendo las moléculas del lípido a la temperatura de $20^{\circ} \mathrm{C}$. Es observable que, independientemente de la temperatura de operación, la monocapa posee una estructura característica de líquido expandido. Es decir, las interacciones entre las cadenas hidrocarbonadas de las moléculas que constituyen la monocapa son prácticamente insensibles a las variaciones de la temperatura del sistema, lo cual puede ser lógico, si se tiene en cuenta que con esta conformación, y como se deduce de los valores de áreas moleculares registradas, la separación entre las citadas moléculas ha de ser significativamente mayor. Como consecuencia de ello se puede extraer una conclusión de interés práctico: las monocapas de ácido oleico esparcidas sobre agua desionizada se caracterizan por su baja compresibilidad.

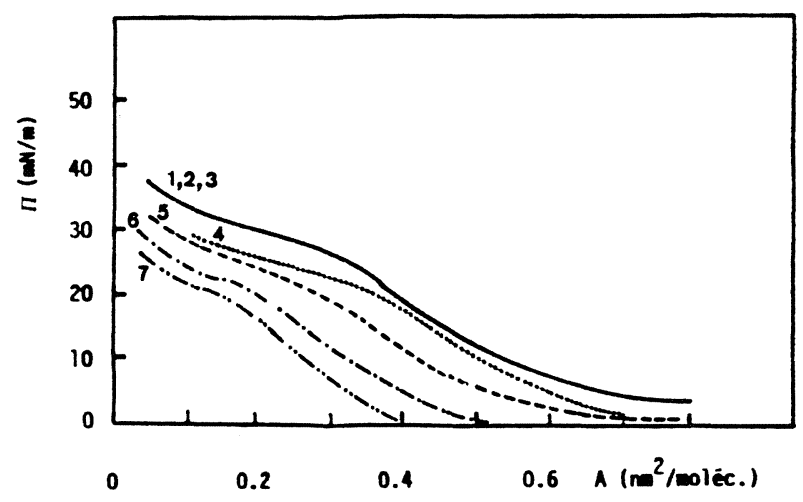

Figura 7

Isotermas de compresión presión superficial-área por molécula de ácido oleico esparcido sobre agua desionizada.

Temperaturas: $1: 5^{\circ} \mathrm{C}, 2: 10^{\circ} \mathrm{C}, 3: 15^{\circ} \mathrm{C}, 4: 20^{\circ} \mathrm{C}, 5: 25^{\circ} \mathrm{C}$, $6: 35^{\circ} \mathrm{C}, \quad 7: 40^{\circ} \mathrm{C}$.

Desde otro punto de vista, es admisible pensar que el ácido oleico posee, en las condiciones de experimentación, una cierta capacidad de difusión hacia la subfase según se deduce de los desplazamientos, hacia menores requerimientos de área molecular, de las isotermas registradas a temperaturas crecientes. No obstante, la pérdida de moléculas de la monocapa que puede deducirse de la representación de la figura 7 puede ser errónea al existir una doble incidencia de la temperatura. En efecto, es observable que al modificarse la temperatura a la cual se realiza la extensión de las moléculas de lípido sobre la interfase se produce una variación de la línea base, según se expone en la Tabla VI. Por lo tanto, si se efectúa una traslación de las isotermas, según los valores en que se modifica la línea base, las diferencias entre ellas quedan significativamente reducidas, aunque la opinión de existencia de inestabilidad en la monocapa, por disolución de sus moléculas, permanece siendo válida. Finalmente, debe indicarse la existencia de una reducción en la presión de colapso de la monocapa al aumentar la temperatura, un fenómeno que se ha observado en experiencias anteriores con otros lípidos, y se ha asociado a la disolución del mismo en la subfase (23) (24).

Tabla VI.

Presión de colapso y reducción de presión a expansión infinita $\left(\Delta \pi_{\mathrm{o}}\right)$, en monocapas de ácido oleico esparcidas sobre agua desionizada.

\begin{tabular}{|ccc|}
\hline$\frac{T}{\left.{ }^{\circ} \mathrm{C}\right)}$ & $\frac{\pi_{\mathrm{c}}(\mathrm{mN} / \mathrm{m})}{39 \cdot 5}$ & $\frac{\Delta \pi_{\circ}(\mathrm{mN} / \mathrm{m})}{2}$ \\
\cline { 3 - 3 } 10 & $39 \cdot 5$ & 4 \\
15 & $39 \cdot 5$ & 4 \\
20 & $33 \cdot 0$ & 0 \\
25 & $33 \cdot 0$ & 0.5 \\
35 & $31 \cdot 0$ & -0.8 \\
40 & 28.4 & -1.4 \\
\hline
\end{tabular}

Monocapas de ácido oleico esparcidas sobre disolución acuosa de etanol

Las experiencias con el ácido oleico se completan esparciendo el mismo sobre una disolución de etanol a $0,5 \mathrm{~mol} / \mathrm{L}$. Los resultados obtenidos se representan en la figura 8 . Comparadas con las monocapas del mismo lípido extendido sobre agua desionizada, discutidas previamente, la presencia de etanol en la subfase potencia la expansión de la molécula de la monocapa y su disolución.

\subsection{Consideraciones de carácter práctico}

Mediante las isotermas $\pi$-A de una monocapa es posible extraer información sobre dos características de la misma de importancia desde un punto de vista de aplicación de estas sustancias como estabilizadoras de emulsiones. Estas características son la solubilidad y la estructura que adoptan las moléculas en la película interfacial, las cuales se pueden optimizar en función de la composición de la subfase, de las moléculas que constituyen la monocapa y de la temperatura.

La solubilidad de una monocapa de un emulsionante alimentario puede establecer la fracción 


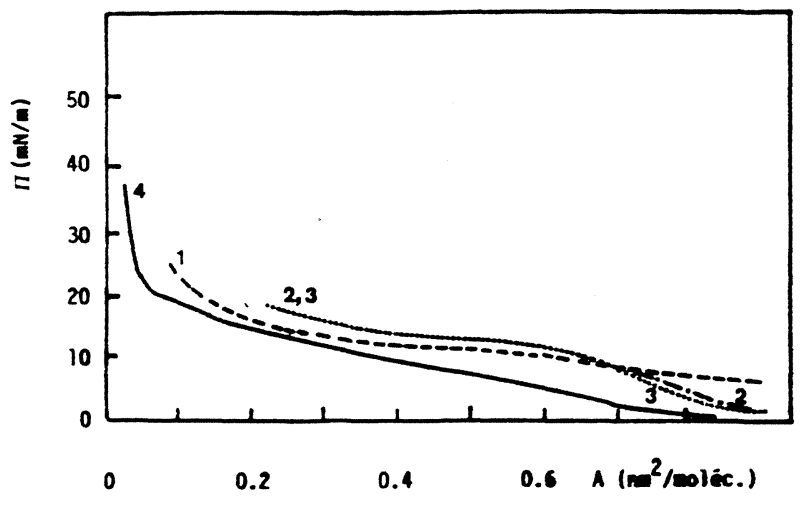

Figura 8.

Isotermas de compresión presión superficial-área por molécula de ácido oleico esparcido sobre disolución acuosa de etanol a $0.5 \mathrm{~mol} / \mathrm{L}$.

Temperaturas: $1: 5^{\circ} \mathrm{C}$ (Primera compresión), 2: $5^{\circ} \mathrm{C}$

(Segunda compresión), 3: $10^{\circ} \mathrm{C}, 4: 25^{\circ} \mathrm{C}$.

de las cantidades permitidas del mismo en una formulación alimentaria que es eficaz en el proceso. La estabilidad de una monocapa de ácido graso se puede incrementar, en general, si: a) Aumenta la longitud de la cadena hidrocarbonada del ácido graso o existen insaturaciones en la misma, b) Disminuye la temperatura del sistema, c) Disminuye el número de grupos alcohólicos en la molécula de soluto que constituye la subfase, d) Disminuye, para un mismo soluto, su concentración, en la subfase.

La estructura de la monocapa ofrece una información valiosa para la aplicación de un aditivo alimentario, por su relación con la estructura que adopta en el seno de la fase y con las características reológicas y compresibilidad de la monocapa. Estos factores inciden durante las etapas del procesado del alimento y sobre las propiedades del producto final. Según las experiencias realizadas, las monocapas de ácidos grasos esparcidas sobre medios acuosos que contienen grupos alcohólicos presentan un polimorfismo estructural que se manifiesta mediante las siguientes transiciones de fases:

\section{SOLIDA $\rightarrow$ LIQUIDA CONDENSADA $\rightarrow$ $\rightarrow$ LIQUIDA EXPANDIDA $\rightarrow$ GASEOSA.}

En general, los mismos factores que hacen disminuir la estabilidad de la monocapa pueden favorecer las transiciones de fases en el orden indicado.

\section{BIBLIOGRAFIA}

1. Boyd, J. V.; Parkinson, C. and Sherman, P.- "Factors affecting emulsions stability and HLB (hydrophilic-liophilic balance) concept ${ }^{n}$.- J. Colloid Interface Sci. 41 (1972) 359370 .

2. Larsson, K.- "Stability of emulsions formed by polar lipids".Prog. Chem. Fats Other Lipids. 16 (1978) 163-169.

3. Dickinson, E. and Stainsby, G.- "Progress in the formulation of food emulsions and foams". - Food Technol. 41 (1987) 74-81.

4. Krog, N. J.; Riison, T. H. and Larsson, K.- "Applications in the Food Industry" en "Encyclopedia of Emulsion Technology".- Becher, P. (Ed.). Dekker, New York, 1985.

5. Miyano, K.; Abraham, B.; Katterson, J. and Xu, S.- "The phases of insoluble monolayers: comparison between the surface pressure-molecular area diagram and the shear modules measurements".- J. Chem. Phys. 77 (1983) 47764777.

6. Buhaenko, M. R.; Grundy, M. J.; Richardson, R. M.- "Surface rheology of spread monolayers".- Thin Solid Film. 159 (1988) 171-189.

7. Davies, J. T. and Rideal, E. K.- "Interfacial Phenomena".Academic Press 2nd. Ed. London, 1963.

8. Gaines, G. L.- "Insoluble Monolayers at Liquid-Gas Interfaces".- Interscience Publ. New York, 1966.

9. Gershfeld, N.- "Physical chemistry of lipids film at fluid interfaces".- Ann. Rev. Phys. Chem. 27 (1976) 349-368.

10 Garfias, F.- "Solid monolayers at the water-gas interface".J. Phys. Chem. 83 (1979) 3126-3130.

11. Gershfeld, N.- "Intermolecular energies in condensed, lipid monolayers on water".- J. Colloid Interface Sci. 32 (1970) 167-172.

12. Langmuir, I.- J. Chem. Phys. 1 (1933) 756. Tomado de Breton, M.- "Formation and possible applications of polymeric Langmuir-Blodgett films. A review".- J. Macromol. Sci. Rev. Macromol. Chem. C. 21 (1981) 61-87.

13. Langmuir, I.- J. Chem. Rev. 6 (1929) 451. Tomado de referencia (12).

14. Caillé, A.; Pink, D.; de Vertenil, F. and Zuckermann, M. J."Theoretical models for quasi 2 D mesomorphic monolayers and membrane bilayers".- Can. J. Phys. 58 (1980) 581 611.

15. Beell, G.; Combs, L. and Dunne, L.- "Theory of cooperative phenomena in lipid systems ${ }^{n}$.- Chem. Rev. 81 (1981) 15-48.

16. Nagle, J. F.- "Theory of the main lipid bilayer transition".Ann. Rev. Phys. Chem. 31 (1980) 157-195.

17. Zuckermann, M. J.; Pink, D. A.; Costas, M. and Sanctuary, B. C.- "A theoretical model for phase transitions in lipid monolayers".- J. Chem. Phys. 76 (1982) 4206-4216.

18. Firpo, J. L.; Legre, J. P.; Bois, A. G. and Baret, J. F."Equilibrium and non-equilibrium critical behavior of amphiphilic monolayers at the LE-LC transition: a model with broken symmetry and melted chain packing".- J. Chem. Phys. 81 (1984) 113-120.

19. Baret, J. F.; Bois, A. G.; Dupin, J. J. and Firpo, J. L.- "The liquid expanded and liquid condensed phases in amphiphile monolayers are separated by a second order transition".- J. Colloid Interface Sci. 86 (1982) 370-376. 
20. Marcelja, S.- "Chain ordering in liquid crystals. II. Structure of bilayer membranes".- Biochem. Biophys. Acta 367 (1974) 165-176.

21. Caillé, A.- "Equilibre de phases dans une couche monomoléculaire de molécules semi-flexibles".- Can. J. Phys. 52 (1974) 389-846.

22. Pekalski, A.- "Phase diagrams for a model of a lipid monolayern.- Eur. Biophys. J. 16 (1988) 39-44.

23. Rodríguez Patino, J. M.; de la Fuente Feria, J. and Gómez Herrera, C.- "Monocapas de ácidos grasos. I. Acido esteárico sobre disoluciones acuosas de etanoln.- Grasas y Aceites 42 (1991) 114-120.

24. Rodríguez Patino, J. M.; de la Fuente Feria, J. and Gómez Herrera, C.- "Monocapas de ácidos grasos. II. Acido esteárico sobre disoluciones acuosas que contienen grupos funcionales alcohólicos".- Grasas y Aceites 42 (1991) 220-229.

25. Harkins, W. A.- "The Physical Chemistry of Surface Films".Reinhold, New York, 1952.

26. Adamson, A. W.- "Physical Chemistry of Surfaces".- Ed. John Wiley and Sons, New York, 1976

27. Ter Minassian-Saraga, L.- "The study of adsorption and desorption at liquid surfaces. I. Study of the surface film of a soluble substance".- J. Chem. Phys. 52 (1955) 80-94.

28. Gershfeld, N.- "The liquid condensed/liquid expanded transition in lipid films: a critical analysis of the film balance experiments".- J. Colloid Interface Sci. 58 (1982) 28-40.

29. Bilkadi, Z. and Neuman, R.- "Effect of humidity on desorption of fatty acid monolayers at constant arean.- J. Colloid Interface Sci. 82 (1981) 480-489.

30. Dickinson, E.- "Effect of atractive forces on monolayer permeation and evaporation resistance".- J. Chem. Soc. Faraday Trans. II. 74 (1978) 821-825.

(Recibido: Noviembre 1990) 